\title{
A New Approach to Integrate The RFID in the Internet of Things Using The MQTT Protocol and 6lo-RFID Framwork
}

\author{
Y.Mouzouna $^{1}$, M.Taouzari ${ }^{2}$,A. Mouhsen ${ }^{1}$, S.Benalla ${ }^{2}$, J.Elaoufi $^{1}$, H. Nasraoui ${ }^{1}$
}

Mouzouna.youssef@gmail.com,med.taouzari@gmail.com,mouhsen_ahmed@yahoo.fr

\author{
1 Laboratory IMII, Faculty of Science and technical Settat, Hassan 1st University \\ 2 Laboratory LISA, National School of Applied Sciences, ENSA Berrechid, Hassan 1st University
}

\begin{abstract}
The RFID Tag evolution from legacy passive Tag to smart Tag with sensing capacity plays a vital role in a smart environment especially in the internet of things, but their integration in this field still meet several difficulties. In this paper, we present a novel approach to integrate the RFID smart and legacy Tag in the internet of things using the publish/subscribe pattern with the MQTT (Message Queueing Telemetry Transport) protocol and a proposed 6lo-RFID framework. This approach allows integrating an RFID system continuously to the internet of things and reporting event in real time.
\end{abstract}

Keywords: RFID, Internet of Things, IoT, MQTT, Broker, 6lo-RFID Framework.

\section{Introduction}

Radio Frequency Identification (RFID) technology has experienced a continuous evolution in the last decade, by moving from simple transponders for tracking purposes to sensor-equipped smart Tags, which are able to implement sensing and processing functions [1] [2] [3]. Smart Tags have the potential to substitute or complement existing wireless sensor networks (WSNs) due to their tiny size, low power, and very cost-effective identification mechanisms. They may bring undisputed benefits to various IoT application scenarios, where networked Tags with sensing and computing capabilities create smart environments, such as in enhanced supply chain, factory automation, smart-home, smart-city and personal healthcare scenarios [3]. However, to fully exploit the potential offered by Smart RFID Tag, it becomes important to integrate them to the internet of things to enabling them to be accessible from and to communicate with any other networked devices in the Internet to create a fully smart environment.

The Internet Engineering Task Force (IETF) is driving the standardization of IPv6-based protocols for the IoT. The IPv6 over Networks of Resource-constrained Nodes (6lo) working Group (WG) [4] is currently enabling IPv6 connectivity for a variety of link-layer technologies in constrained node networks with limited power, memory and processing resources, such as Bluetooth Low Energy (BLE) [5] and Near Field Communication (NFC). The 6lo standardization effort has not yet addressed UHF RFID among the link-layer technologies of 
interest, albeit the potential of modern smart Tags to be included as fully-fledged components in the IoT.

In this article, we design an IPv6-based internetworking solution, which natively enables smart RFID Tags and legacy tags to communicate directly with any other devices connected to the Internet. Kevin Ashton, the co-founder of the MIT's Auto-ID Center, which developed the global standard for RFID, introduces the term IoT and other sensors, describing the Internet of Things as a system where the Internet is connected to the physical world through ubiquitous sensors [6]. The Internet of Things can be defined as a dynamically changeable and selfconfigurable, global network infrastructure having special characteristics based on interpretable communication protocols. In this infrastructure, physical and virtual "things" are intelligent and have unique identities, physical attributes, and are seamlessly integrated into the information network [7].

This paper describes an innovative solution based on the publish/subscribe pattern and 6lo-RFID framework to integrate the RFID Tag on the internet of things. this paper is organized as follow: a related works is presented in section 2, in section 3 the proposed approach is presented, in section 4 the 6lo-RFID framework for MQTT is described. In section 5 the communication pattern is presented and a conclusion and recommendations are developed in section 6 .

\section{Related Works}

The integration of RFID technology in the IoT domain still requires further attention. There are many works in the literature focusing on the integration of RFID in the IoT Network by leveraging the IP stack, but their goals are only partially achieved and, typically, they lack flexibility. In [8] the concept of virtual endpoint was introduced, in [9] a proposed CoAPcompliant Solution for Efficient Inclusion of RFID in the Internet of Things is developed. In [10] an ultralow-power hybrid-sensing network composed of 6LoWPAN nodes that integrate UHF RFID functionalities is designed for e-Health applications. This solution requires that hybrid RFID Gen2 Tags are equipped with an additional IEEE 802.15.4 interface to exchange 6LoWPAN-compliant packets, thus increasing both device complexity and production cost. In [11] A Framework for Full Integration of Smart UHF RFID Tags into the Internet of Things was introduced by designing and testing a unified IPv6-based framework to access and manipulate RFID Tags' resources by means of the standard CoAP. [12] Introduces a semi passive, reconfigurable UHF RF identification (RFID) sensing Tag operating as the generic sensing platform (GSP) and the sensing nodes of the future Internet of Things (IoT). In [13] a new design and implement for integrating RFID to the Internet of Things where RFID Tags' resources are accessible via a reader acting as a CoAP proxy. The solution is backward compatible with legacy RFID systems, although it enforces the reader to interpret CoAP application requests and consequently manage Tags' resources. However, flexibility and scalability are limited by vendor lock-in issues and by the need for reader's firmware updates whenever Tags have new resources to offer. All this work uses the CoAP protocol, which is very useful when we need to access or update RFID Tag data. However, the CoAP protocol is not useful for RFID applications with requirements for continuously reporting events of RFID Tag reads (EPC codes) from many different origins. In [14] the RFIPv6 architecture makes a global access to the information stored in the EPCglobal platform, but with no real-time communications with RFID Tags. 


\section{Proposed approach}

In our approach, we used the publish/subscribe pattern to integrate the RFID Tag in the internet of things. The protocol used is the MQTT (Message Queueing Telemetry Transport). The MQTT is a many-to-many communication protocol, which allows multiple clients to exchange messages using a central broker who is in charge of routing incoming messages based on published services "see Figure.1". MQTT is more matured and stable than CoAP. It is best used when a node sends data at irregular intervals of time i.e. event driven system which is good for RFID applications with requirements for continuously reporting events of RFID Tag reads (EPC codes) from many different origins [15]. MQTT is an asynchronous messaging protocol.

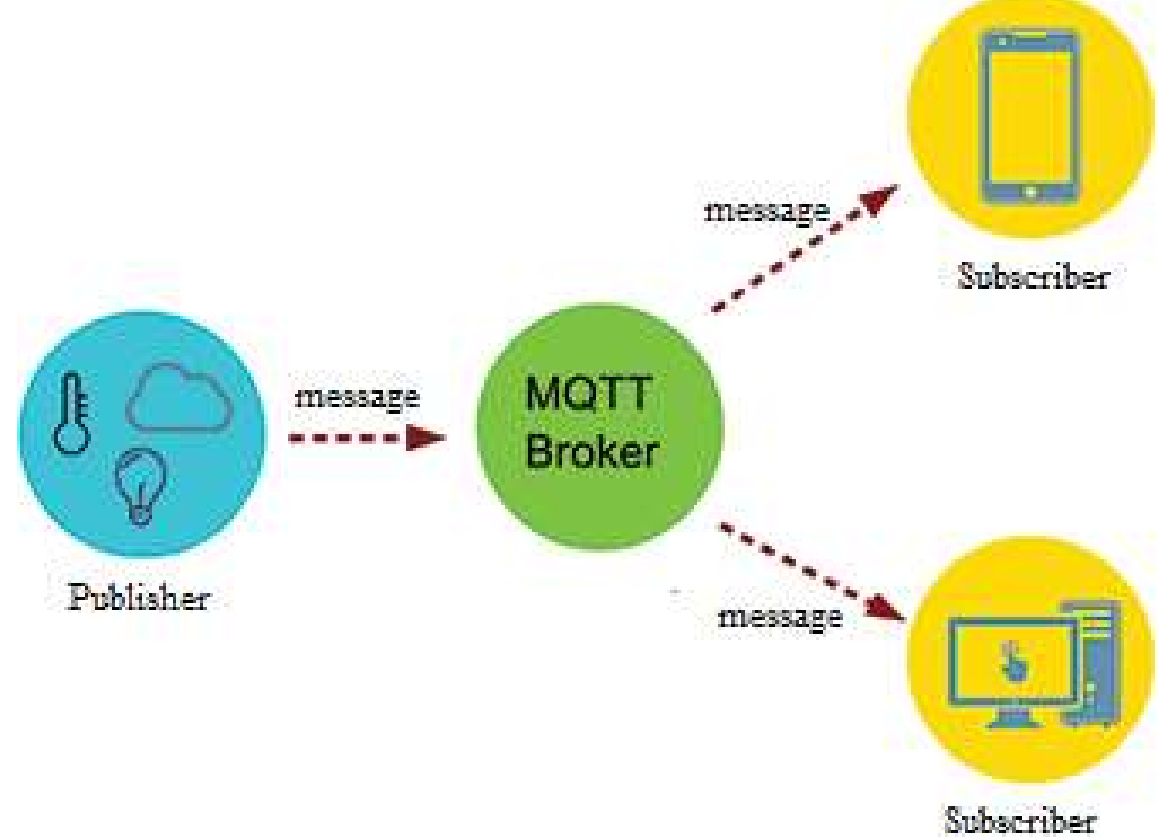

Fig. 1. The Principe of the fonctionement of the MQTT Protocol

The MQTT protocol is designed for light Machine-to-Machine communications in constrained networks [2] or high latency networks [3]. The MQTT Publisher publishes messages to an MQTT broker, which are subscribed by other clients (Subscriber) or may be retained for future subscriptions. Each message is published at an address, known as Topic [16]. Customers can subscribe to several Topics and receive each message posted to each Topic. MQTT is a binary protocol and normally requires a fixed 2-byte header with a small message payload of up to 256 MB [17]. It uses TCP as transport protocol and hence perfect for scenarios where connectivity is required all through, also it use TLS / SSL for security [17]. Thus, the communication between the client and the broker is a connection oriented. Another great feature of MQTT is its three levels of Quality of Service (QoS) for reliable delivery of messages [18].

The proposed system consists essentially of smart Tag to send their EPC and sensing data to the reader and legacy Tags their EPC to the reader. The reader will send this data to an MQTT broker or receives data from client to write them on the Tag memory; The MQTT broker can be 
any hardware with a specific software for example a raspberry pi with a software like Mosquitto MQ. Many different brokers already support MQTT, such as ActiveMQ, Apollo, HiveMQ, IBM Message Sight, JoramMQ, RabbitMQ, MosquitoMQ and VerneMQ. The RFID reader will play the role of both a publisher by publishing continuously the data collected from the legacy Tags and smart Tags, and will also play the role of subscriber to receive data form another publisher. The clients or others devices in the network will play the role of the subscriber to read data from the Tags published in the broker in near real time and also the role of a publisher to send data to the Tags as depicted in " Figure.2". The messages will be published in Topics in the MQTT broker, the name of each Topic will be the EPC of the Tag of each Tag.

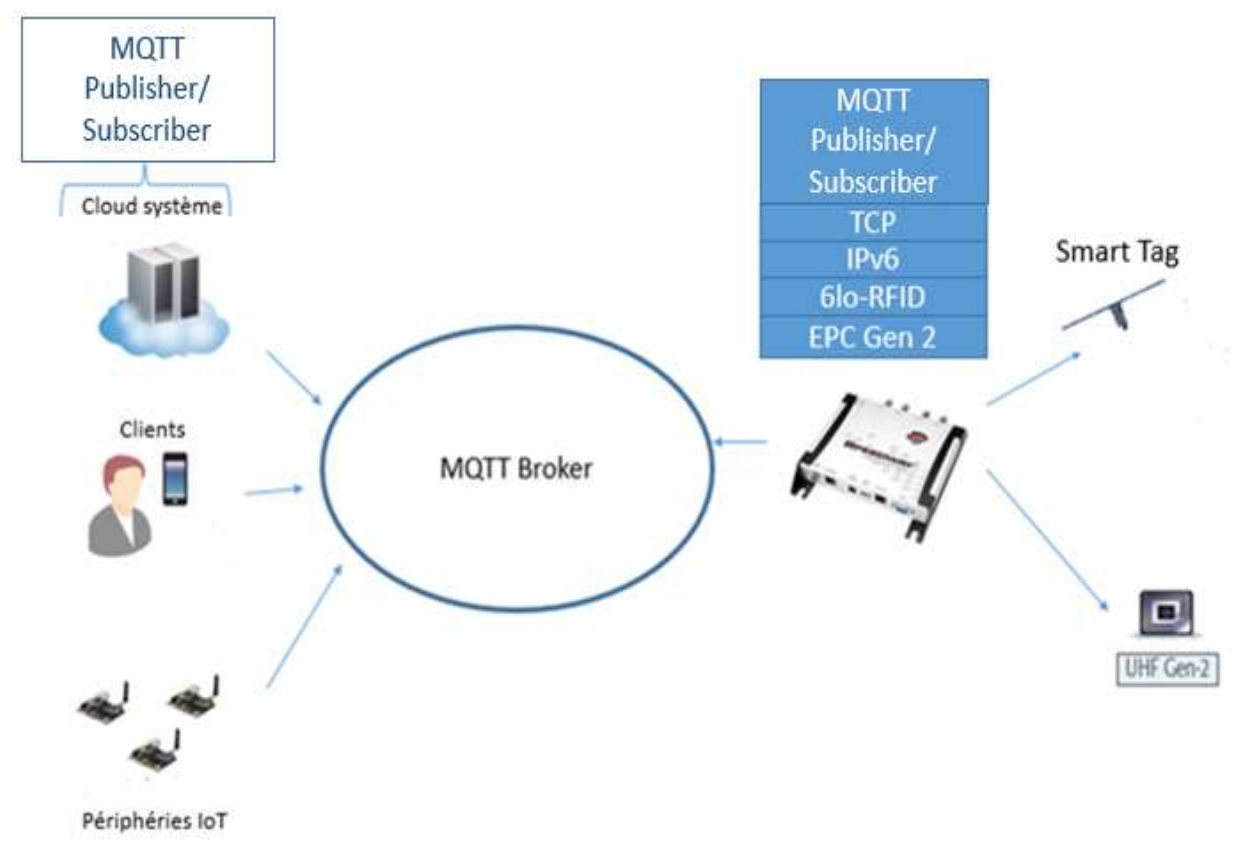

Fig. 2. The proposed system based on publish/subscribe pattern

\section{6lo-RFID Framework}

In the proposed system, RFID smart Tag will communicate by using IPv6 Address. For that purpose, we developed a framework for using the MQTT protocol and the Ipv6 for addressing each Tag. To allow the exchange of IPv6 packets generated by (or directed to) a given Tag, we propose a new organization of the user memory of the RFID Tag (UM), shown in Table. 1. According to the EPCglobal Tag Data Standard, the first eight bits of Unified Messaging contain the DSFID (Data Storage Format IDentifier) field, which specifies the format of the rest of the Unified Messaging store. This field includes the data format to indicate which data system predominates in the MU. Clearly, a new data format code must be associated with IPv6-based 
RFID communications; this maintains compatibility with other RFID data standards. The following bits are reserved for future use (RFU) to manage the protocol version, priority management, or congestion management of the queue. Since the read and write commands operate on 16-bit word units, we propose to store the 6lo-RFID frame starting with the second 16-bit word of the MU to avoid unnecessary read / write of the field. The following fields are included in the 6lo-RFID header ( 2 to 4 bytes) 6lo-RFID header " see Figure. 3".

Table 1. The Proposed 6lo-RFID packet format

\begin{tabular}{|c|c|c|c|c|c|c|c|}
\hline DSFID & RFU & $\begin{array}{c}\text { 6lo-RFID } \\
\text { Header }\end{array}$ & $\begin{array}{c}\text { IPv6 } \\
\text { Header }\end{array}$ & $\begin{array}{c}\text { TCP } \\
\text { Header }\end{array}$ & $\begin{array}{c}\text { MQTT } \\
\text { Header }\end{array}$ & Payload & FCS \\
\hline
\end{tabular}

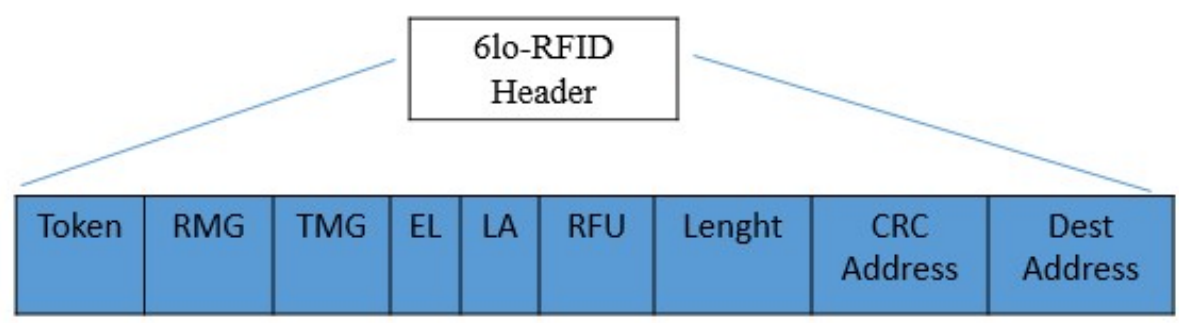

Fig. 3. 6lo-RFID Header Packet Format

Token (1 bit): Used to guarantee exclusivity in the write operation and, thus, manage simultaneous access to the UM of the Tag for the reader's RFID reader and sensor. Its value 0 allows access to the RFID reader to the MU, while the value 1 allows the access of the sensor to the UM. To avoid blocking, the reader resets the UM to start a new exchange of information if the sensor does not clear the token bit before an optimal time. Reader Message (RMG) (1 bit): Indicates if the reader has written a message into the Tag. The Tag subsequently clears this bit after reading the relevant reader message. Tag Message (TMG) (1 bit): Indicates if the Tag has inserted a message in the UM. The reader clears this bit after reading the Tag message. Extended Length (EL) (1 bit): If the packet length is more than 255 bytes and the UM contains it, then this bit must be set to one to use an Extended Length field of 16 bits. Local Addressing (LA) (1 bit): Its value 1 indicates that a local addressing strategy is performed. In this case, the header includes source and destination addresses (more details are given in the following). (RFU) Reserved for Future Use (3 bits). Length (8-16 bits): Indicates the number of bytes that compose the 6lo-RFID packet (the field allows to exchange up to 65535 byte-packets). A frame check sequence (FCS) of 32 bits is added at the tail of the 6lo-RFID frame to detect tampering or inconsistencies due to errors or partial writing of the UM. Considering the limited frame size, header compression is highly advisable to efficiently exchange data. To save valuable bits in the memory, The IPv6 And TCP headers shall be compressed according to the standard encoding 
schemes defined in IETF RFC 6282 [4]. Since IPv6 defines a maximum transmission unit (MTU) of 1280 bytes, the 6lo-RFID layer must also implement fragmentation and reassembly functionality, as defined in RFC4944 [4], when the UM is not large enough to contain the whole IPv6 packet. 


\section{Communication Pattern}

The proposed approach is a master-slave approach to accessing the UM and managing the reader-Tag interaction, where the reader acts as a master and controls the flow of data from / to all RFID Tags in its coverage area. The proposed solution is compatible with the Gen-2 protocol because it can easily exploit the already defined Gen- 2 commands. The reader initiates the label singulation phase by specifying the EPC code of the desired label in a SELECT command, so that only the label with the specified EPC can participate in the inventory phase and the collisions, communication between two Tags is done after reading of each tag in its area. Then, the Writing and Reading commands respectively allow the transfer of data in the downlink and uplink directions. All these data collected by the reader are published in the broker. This operation is carried out continuously in order to evaluate any event in the RFID system. The communication pattern is described "in the Figure.4".

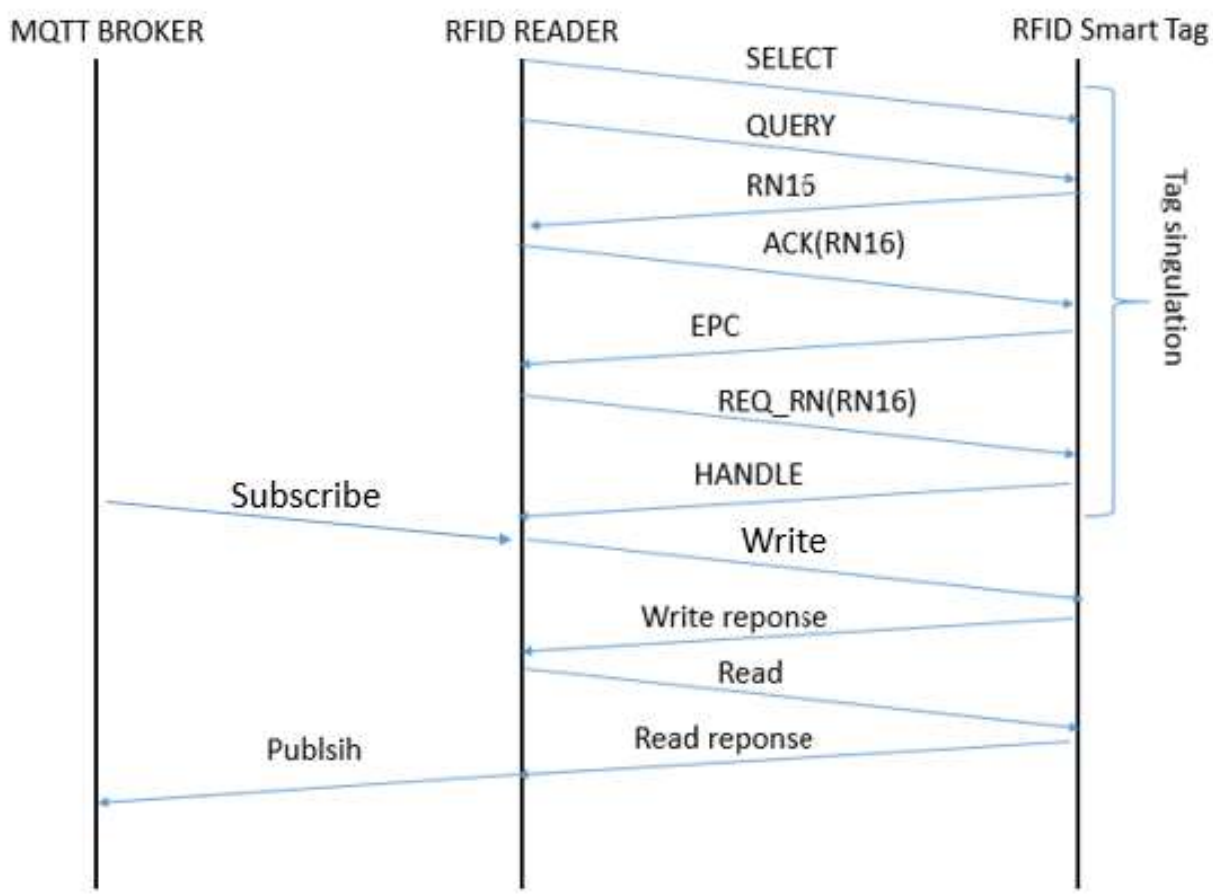

Fig. 4. The proposed system based on publish/subscribe pattern 


\section{Conclusion}

In this article, a novel approach based on IPv6 framework and publish/subscribe pattern using MQTT protocol has been designed to transparently integrate RFID systems into the IoT domain and enable IPv6 communication and networking over UHF RFID smart Tags. This approach uses a new packet format of data stored in the UM of the RFID Tag called 6lo-RFID. This framework includes and IPv6 header, TCP header, MQTT header. This approach is compatible with the standard EPCglobal Gen-2 protocol which make it flexible to use. 


\section{References}

[1] J. Smith, A. Sample, P. Powledge, S. Roy et A. Mamishev, "A wirelessly-powered platform for sensing and computation," Ubiquitous Computing, Lecture Notes in Computer Science; Springer: Orange County, CA, USA,, Vol. 4206, pp. 495-506, 2006.

[2] D. De Donno, L. Catarinucci et L. Tarricone, ".A battery-assisted sensor-enhanced RFID Tag enabling heterogeneous wireless sensor networks,” IEEE Sens., pp. 1048-1055, 2014.

[3] M. Khan, M. Islam et D. Hai, "Design of a reconfigurable RFID sensing Tag as a generic sensing platform toward the future Internet of Things." IEEE Internet Things, pp. 300-310, 2014.

[4] IETF 6LoWPAN Working Group, https://tools.ietf.org/wg/6lowpan.

[5] J. Nieminen ; C. Gomez, "Networking Solutions for Connecting Bluetooth Low Energy Enabled Machines to the Internet of Things," IEEE Network, Vol. 28, No. 16, pp. 83-90, 2014.

[6] Kevin Ashton, "That 'Internet of Things' Thing, RFID Jouranl, 2009.

[7] F. Hussein, Internet of things: Building blocks and business models, Canada,: springer, 2017.

[8] Jara AJ, Moreno-Sanchez P, Skarmeta AF, "IPv6 Addressing Proxy: Mapping Native Addressing from Legacy Technologies and Devices to the Internet of Things (IPv6)" Sensors, Vol. 13, No.15, pp. 6687-6712., 2013.

[9] Ivan Farris ; Antonio Lera ; Antonella Molinaro ; Sara Pizzi, "A CoAP-Compliant Solution for Efficient Inclusion of RFID in the Internet of Things" IEEE Global Commun. Conf., pp. 2795-2800., 2014.

[10] L. Catarinucci ; D. de Donno ; L. Mainetti ; L. Palano "An IoT-Aware Architecture for Smart Healthcare Systems” IEEE Internet of Things, Vol. 2, No16, pp. 515-526, 2015.

[11] S. P. Ivan Farris, M. Merenda, A. Molinaro, R. Carotenuto et a. A. Iera, "6lo-RFID: A Framework for Full Integration of Smart UHF RFID Tags into the Internet of Things," IEEE Network, 2017.

[12] S. Islam ; a. D. Ha, "Design of a Reconfigurable figurable RFID Sensing Tag as a Generic Sensing Platform Toward the Future Internet of Things," IEEE Internet of Things , Vol. 1, No.14, pp. 300-310, 2014.

[13] I.Varis; S.Pizzi; A.Molinaro "Design and Implementation of a CoAP-Compliant Solution for RFID Inclusion in the Internet of Things" J.Sens.Actuator Netw,Vol.5, No.4, 2016.

[14] Yao-Chung Chang; Jiann-Liang Chen; Yu-Shan Lin ; Shi-Ming Wan "RFIPv6 - A Novel IPv6EPC Bridge Mechanism” International Conference on Consumer Electronics IEEE, 2008

[15] F.Valente; A. Neto "Inteligent Steel Inventory Tracking with IoT/RFID" IEEE International Conference on RFID Technology \& Application (RFID-TA) ,2017.

[16] Youngchoon Park, Building an effective IoT ecosystem for your business, Milwaukee, Wisconsin, USA: Springer, 2017.

[17] A. Gilchrist, Industry 4.0: The industrial internet of things, Apress, 2016.

[18] Bhattacharyya, «Lightweight internet protocols for web enablement of sensors using constrained gateway devices,» IEEE International Conference on Computing, Networking and Communications (ICNC), pp. 334-340, 2013. 